\title{
Isospin symmetry breaking and the neutron-proton mass difference
}

\author{
N.F. Nasrallah \\ August 29, 2018 \\ Lebanese University, Faculty of Science, Tripoli, Lebanon
}

\begin{abstract}
QCD sum rules using polynomial kernels are used to evaluate the strong part of the proton-neutron mass difference $\delta M_{n p}$ in a model independent fashion. The result for the mass difference turns out to depend sensitively on the value of the four quark condensate $\left\langle(\bar{q} q)^{2}\right\rangle$ and reproduces the experimental value of $\delta M_{n p}$ for $\left\langle(\bar{q} q)^{2}\right\rangle \sim 2\langle\bar{q} q\rangle^{2}$.
\end{abstract}




\section{Introduction}

The QCD sum rule method introduced by Shifman, Vainshtein and Zakharov [1] has extended the applicability of QCD far beyond simple perturbation theory. The method was adapted to the case of nucleons by Ioffe [2] and independently by Chung, Dosch, Kremer and Schall [3. These authors showed how to approach one of the fundamental problems of hadronic physics, the calculation of the baryon masses from the Lagrangian and the vacuum condensates of QCD.

Several authors [4] undertook the study of the neutron-proton mass difference $\delta M_{n p}$ using the external field method of Ioffe and Smilga 2]. These authors use Borel (Laplace) transforms of correlators in order to suppress the unknown contributions of the continua which appear in dispersion integrals.

I present here a different approach which uses instead as kernels in the dispersion integrals simple polynomials [5] the coefficients of which are determined by the general shape of the physical spectrum and which, as will be shown, offer stronger damping of the unknown parts of the spectrum in addititon to better stability.

\section{The Calculation}

It has been known since the early days of QCD that the mass splitting of hadrons in an isospin multiplet arises from two sources: virtual photon exchange, which can be related to virtual photon scattering by the Cottingham formula [6] and isospin symmetry breaking which arises from the difference between the masses of the light quarks $m_{u}$ and $m_{d}$ as well as the difference between the light quark condensates $\langle\bar{u} u\rangle$ and $\langle\bar{d} d\rangle$.

Let us start by a reminder of how electromagnetic and strong parts of the mass splittings arise [9]

The quark mass term in the QCD Hamiltonian density reads

$$
H(x)=m_{u} \bar{u} u+m_{d} \bar{d} d+\ldots
$$

The effect of turning on electromagnetism is described by the additional term

$$
H_{e m}(x)=\frac{e^{2}}{2(2 \pi)^{4}} \int \frac{d^{4} q}{q^{2}} \int d^{4} x e^{i q x} T j_{\mu}(x) j_{\mu}(0)
$$

which accounts for virtual photon exchange.

$$
j_{\mu}=\frac{1}{3} \bar{u} \gamma_{\mu} u-\frac{2}{3} \bar{d} \gamma_{\mu} d
$$

is the electromagnetic current expressed in terms of quark fields.

Following Cottingham[6] eq. (11) can be transformed into 


$$
\begin{aligned}
H_{e m}(0) & =\frac{e^{2}}{8 \pi^{2}} \int_{0}^{\infty} \frac{d Q^{2}}{Q^{2}} \int_{-Q}^{Q} d q_{0} \sqrt{Q^{2}-q_{0}^{2}} \cdot i \int d^{4} x e^{i q x} T j_{\mu}(x) j_{\mu}(0) \\
& =\frac{e^{2}}{8 \pi^{2}} \int_{0}^{\lambda} \frac{d Q^{2}}{Q^{2}} \int_{-Q}^{Q} d q_{0} \sqrt{Q^{2}-q_{0}^{2}} \cdot i \int d^{4} x e^{i q x} T j_{\mu}(x) j_{\mu}(0) \\
& +\frac{e^{2}}{8 \pi^{2}} \int_{\lambda}^{\infty} \frac{d Q^{2}}{Q^{2}} \int_{-Q}^{Q} d q_{0} \sqrt{Q^{2}-q_{0}^{2}} \cdot i \int d^{4} x e^{i q x} T j_{\mu}(x) j_{\mu}(0)
\end{aligned}
$$

where $Q^{2}=-q^{2}$ and $\lambda$ is a divider between small and large values of $Q^{2}$.

Matrix elements of the low energy contribution to eq. (44) can be obtained from experimental observation, the insertion of intermediate states, etc., this term we call $H_{\text {coul. }}^{\lambda}(0)$, the "Coulomb" part. In the (divergent) high energy part we can use the operator product expansion (OPE) of the electromagnetic currents with the result

$$
H_{e m}(0)=H_{\text {coul }}^{\lambda}(0)+\frac{e^{2}}{6 \pi^{2}} \ln \frac{\Lambda^{2}}{\lambda^{2}} m_{d} \bar{d} d-\frac{e^{2}}{24 \pi^{2}} \ln \frac{\Lambda^{2}}{\lambda^{2}} m_{u} \bar{u} u+\ldots
$$

with $\Lambda$ an ultraviolet cutoff.

When this is added to expression (11) the divergent terms are absorbed in the renormalized masses and the Hamiltonian density takes the form

$$
H(0)=H_{\text {coul }}(0)+\frac{\left(m_{u}+m_{d}\right)}{2}(\bar{u} u+\bar{d} d)+\frac{\left(m_{u}-m_{d}\right)}{2}(\bar{u} u-\bar{d} d)
$$

The first and last terms in the equation above break isospin symmetry and contribute to the neutron-proton mass difference $\delta M_{n p}$.

$$
\delta M_{n p}=\left\langle p\left|H_{\text {coul }}(0)\right| p\right\rangle+\frac{\delta m}{2 M_{p}}\left\langle p\left|u_{3}\right| p\right\rangle=\delta M_{\text {coul }}+\delta M_{q}
$$

with

$$
\begin{gathered}
\delta m=m_{d}-m_{u} \\
u_{3}=\bar{u} u-\bar{d} d
\end{gathered}
$$

This separation depends of course on the value of the divider $\lambda$ but this dependence is extremely weak: the relative change in the value of $\delta M_{q}$ for two values $\lambda_{1}$ and $\lambda_{2}$ is

$$
\sim 1.5 .10^{-3} \ln \frac{\lambda_{2}^{2}}{\lambda_{1}^{2}}
$$

$\delta M_{\text {coul }}$ has been estimated a long time ago [7], $\delta M_{\text {coul }}=-(.76 \pm .30 \mathrm{MeV})$. A recent evaluation [8] which is here adopted is

$$
\delta M_{\text {coul }}=(-1.30 \pm .50) M e V
$$


The measured value $\delta M_{n p}=1.29 \mathrm{MeV}$ yields then

$$
\left(\delta M_{q}\right)_{\exp }=(2.60 \pm .50) M e V
$$

The theoretical expression is given by eq. (7)

$$
\delta M_{q}=\delta m U
$$

with

$$
U=\left\langle p\left|u_{3}\right| p\right\rangle / 2 M_{p}
$$

The aim of the present calculation is to evaluate $U$ using the asymptotic forms of the various matrix elements given by QCD and the incomplete available knowledge about the nucleon continuum with an emphasis on the stability of the calculation. For this purpose I follow the external field approach of Ioffe and Smilga 22 in which the quarks are coupled to a weak external scalar field $S(x)$ through an additional term to the QCD Lagrangian

$$
\Delta L=-S(x)(\bar{u} u-\bar{d} d)
$$

$S(x)$ can be taken a constant. The correlation function of the nucleon currents in the presence of $S(x)$ is

$$
\Pi(S, q)=i \int d^{4} x e^{i q . x}\left\langle 0\left|T \eta_{p}(x) \overline{\eta_{p}}(0)\right| 0\right\rangle_{S}
$$

where $\eta_{p}(x)$ is the proton interpolating field of Ioffe 2 ]

$$
\eta_{p}(x)=\epsilon_{a b c} U_{a}^{T}(x) C \gamma_{\mu} U_{b}(x) \gamma_{5} \gamma_{\mu} d_{c}(x)
$$

where a, b, c stand for colour indices and $C=-C^{T}$ is the charge conjugation matrix. Lorentz covariance and parity allow the decomposition

$$
\Pi(S, q)=\Pi^{1}(S, q)+\gamma \cdot q \Pi^{q}(S, q)
$$

To first order in $S$ the two invariant functions can be written as

$$
\Pi^{i}(S, q)=\Pi_{0}^{i}\left(q^{2}\right)+S \Pi_{1}^{i}\left(q^{2}\right)
$$

where $\Pi_{0}^{i}$ denote the invariant functions in the absence of the external field and $\Pi_{1}^{i}$ are the linear responses to the latter which, in QCD, can be expressed via the OPE to various vacuum condensates.

The external field will contribute in two different ways: by directly coupling to the nucleon fields which enter in the nucleon current and by polarizing the QCD vacuum. This will introduce a susceptibility $\chi$ which describes the response of the quark condensates to $S$,

$$
\begin{aligned}
& \langle\bar{u} u\rangle_{S}=\langle\bar{u} u\rangle-\chi S\langle\bar{u} u\rangle \\
& \langle\bar{d} d\rangle_{S}=\langle\bar{d} d\rangle+\chi S\langle\bar{d} d\rangle
\end{aligned}
$$


where $\langle\bar{q} q\rangle \equiv\langle 0|\bar{q} q| 0\rangle$

Using eq. (14) one obtains

$$
\chi\langle\bar{u} u\rangle=\frac{i}{2} \int d^{4} x\left\langle 0\left|T u_{3}(x) u_{3}(0)\right| 0\right\rangle
$$

The QCD expression for $\Pi_{1}^{q}(t)$ has been evaluated in [4]

$$
\Pi_{1}^{q}(t)=\left(C_{0} \ln (-t)+\frac{C_{1}}{t}+\frac{C_{2}}{t^{2}}+\ldots\right)
$$

The constants $C_{i}$ are expressed in terms of the quark condensates, the susceptibility $\chi$ and the quark gluon mixed condensate $g_{s} \bar{q} \vec{\sigma} \cdot \vec{G} q$ and its corresponding susceptibility $\chi_{m}$

$$
\begin{aligned}
C_{0} & =\frac{\langle\bar{q} q\rangle}{4 \pi^{2}}, \\
C_{1} & =\frac{4}{3} \chi\left\langle(\bar{q} q)^{2}\right\rangle-\frac{m_{0}^{2}}{24 \pi^{2}}\langle\bar{q} q\rangle, \\
C_{2} & =\left(\chi+\chi_{m}\right) m_{0}^{2}\langle\bar{q} q\rangle^{2} / 6
\end{aligned}
$$

To a good approximation $\chi_{m}=\chi[4$. With the estimate

$$
m_{0}^{2}=\frac{\left\langle g_{S} \bar{q} \vec{\sigma} \cdot \vec{G} q\right\rangle_{0}}{\langle\bar{q} q\rangle} \cong(.80 \pm .20) G e V^{2}
$$

At low energies $\Pi_{1}^{q}(t)$ has double and single isolated poles at the nucleon mass squared as well as a cut on the positive real axis starting at $t_{t h}=\left(M_{p}+m_{\pi}\right)^{2}$

$$
\Pi_{1}^{q}(t)=\frac{-2 \lambda^{2} M_{p} \cdot U}{\left(t-M_{p}^{2}\right)^{2}}+\frac{A}{\left(t-M_{p}^{2}\right)}+\ldots
$$

where $\lambda$ is the coupling of the nucleon to its current

$$
\left\langle 0\left|\eta_{p}\right| p\right\rangle=\lambda U_{p}
$$

In order to relate the residue $U$ to the QCD condensates consider the integral of the product $\Pi_{1}^{q}(t) F(t)$ over a contour $C$ in the complex $t$ plane. The contour $C$ consists of two straight lines parallel to the cut, sandwiching it from above and below, and running from threshold to a value $R$ and a circle of radius $R$ taken large enough to allow the replacementof $\Pi_{1}^{q}(t)$ by its asymptotic form eq. (21) on it.

$F(t)$ is a so far arbitrary entire function. Using next Cauchy's theorem we obtain an expression relating the residue at the pole to an integral over the cut plus an integral over the circle where the QCD expression for the amplitude can be used. The integral over the cut cannot be evaluated as the information on the integrand over the continuum is scarce. We shall use the arbitrariness in the choice of $F(t)$ to minimize this contribution so it can be safely neglected. 
First take

$$
F(t)=\left(1-\frac{t}{M_{p}^{2}}\right) P(t)
$$

in order to eliminate the contribution of the simple pole which represents nucleon to continuum transitions. Cauchy's theorem then yields

$$
2 \lambda^{2} M_{p} U P\left(M_{p}^{2}\right)=\frac{1}{\pi} \int_{t h}^{R} d t F(t) \operatorname{Im} \Pi_{1}^{q}(t)+\frac{1}{2 i \pi} \oint d t F(t)\left(\Pi_{1}^{q}(t)\right)_{Q C D}
$$

The second integral in the expression above runs over the circle of large radius $R$.

The coupling $\lambda$ itself can be obtained from the nucleon mass sum rule [2] [3] [10] using $P(t)$ as an integration kernel with the result

$$
-M_{p} \lambda^{2} P\left(M_{p}^{2}\right)=\frac{1}{\pi} \int_{t h}^{R} d t P(t) \operatorname{Im} \Pi_{2}(t)+\frac{1}{2 i \pi} \oint d t P(t) \Pi_{2}^{Q C D}(t)
$$

with

$$
\begin{aligned}
\Pi_{2}^{Q C D}(t) & =B_{3} t \ln (-t)+\frac{B_{7}}{t}+\frac{B_{9}}{t^{2}}+\ldots \\
B_{3} & =\frac{1}{4 \pi^{2}}\langle\bar{q} q\rangle\left(1+\frac{3}{2} \cdot a\right) \\
B_{7} & =-\frac{1}{12}\langle\bar{q} q\rangle\langle a G G\rangle \\
B_{9} & =4 \pi^{2} \frac{136}{81} a\langle\bar{q} q\rangle^{3}
\end{aligned}
$$

$a=\alpha_{S} / \pi$ is the strong coupling constant. The ratio of eqs. (27) and (28) yields the residue $U$ of interest.

Not enough experimental data is available to allow the evaluation of the first integrals on the r.h.s. of eqs. (27) and (28), only the positions of the $\frac{1}{2}^{+}$and $\frac{1}{2}^{-}$ resonances are known and the background is impossible to model realistically.

The choice of the function $P(t)$ aims at reducing this contribution as much as possible in order to allow its neglect. This is achieved by minimizing the ratio $\mid P(t) / P\left(M_{p}^{2} \mid\right.$ or equivalently $|P(t)|$ over the resonance region. In the vast domain of QCD sum rules the usual choice would be $P(t)=\exp \left(-t / M^{2}\right)$ where the magnitude of $M$ (the Borel mass) determines the strength of the damping of the contribution of the integrals over the continuum. If $M$ is small the damping is good but the contribution of the unknown terms in the QCD asymptotic expansion of the amplitudes increases rapidly. If $M$ increases the contribution of the unknown terms decreases but the damping worsens. An intermediate value of $M$ has to be chosen from stability conditions which are not always met. 
Because the QCD expressions for the amplitude are (except for logarithms ) infinite series in inverse powers of $t, C_{n} / t^{n}$ and because the exponential can be expanded in an infinite series of powers of $t$ of the form $\frac{1}{n !}\left(\frac{t}{M^{2}}\right)^{n}$ the integral over the circle consists of an infinite sum of terms of the form $\frac{C_{n+1}}{n ! M^{2 n}}$ which become important if $M$ is too small. This prompts us to choose for $P(t)$ a polynomial of degree $N$ which involves only a finite number of condensates $\left(C_{1}, C_{2}, . ., C_{N+2}\right)$ resulting from the integral over the circle. In order to limit the uncertainty introduced by the condensates $N$ has to be chosen as small as possible still large enough to provide adequate damping of the continuum. It turns out that a second order polynomial will do the job. This will introduce the unknown condensates $C_{3}$ and $C_{4}$ but,as we shall see, their contribution can be estimated and turns out to be small.

The choice adopted for $P(t)$ stems from the observation of the spectrum of the positive and negative parity nucleon resonances $\mathrm{N}(1440), \mathrm{N}(1535), \mathrm{N}(1650)$ and $\mathrm{N}(1710)$. The position of these resonances imply that the main contribution to the integrals over the nucleon continuum arises from the interval $I: 2.0 \mathrm{GeV}^{2} \precsim t \precsim 3.0 \mathrm{GeV}^{2}$. So let

$$
P(t)=1-a_{1}^{\prime} t-a_{2}^{\prime} t^{2}
$$

The coefficients $a_{1}^{\prime}$ and $a_{2}^{\prime}$ are chosen so as to practically annihilate the contribution of the continuum on the interval $I$, e.g. they can be chosen so as to minimize the integral $\int_{I} d t P(t)^{2}$ which insures the smallness of $|P(t)|$. Another choice, which yields almost identical results, would be to minimize the sum $\sum P(t)^{2}$ at the resonances.

This choice reduces the relative contribution of the integrals over the continua to only a few percent of their initial values and allows their neglect. Numerically

$$
\begin{aligned}
& a_{1}^{\prime}=.807 \mathrm{GeV}^{-2}, \\
& a_{2}^{\prime}=-.160 \mathrm{GeV}^{-4}
\end{aligned}
$$

The relative damping provided by $\mathrm{P}(\mathrm{t})$ at the masses of the nucleon resonances listed above, $\mathrm{P}$ (resonance) $/ \mathrm{P}$ (nucleon) is excellent. This ratio amounts respectively to $.036,-.032,-.027, .019$ which is to be compared to the corresponding values obtained from exponential damping (at $\mathrm{M}^{2}=1.1 \mathrm{GeV}^{2} e . g$.), $.34, .26, .19, .16$.

We have thus

$$
F(t)=1-a_{1} t-a_{2} t^{2}-a_{3} t^{3}
$$

with 


$$
\begin{aligned}
& a_{1}=1.947 \mathrm{GeV}^{-2}, \\
& a_{2}=-1.084 \mathrm{GeV}^{-4}, \\
& a_{3}=.184 \mathrm{GeV}^{-6}
\end{aligned}
$$

If the contributions of the nucleon continua are then neglected eqs. (27) and (28) become

$$
\begin{aligned}
2 \lambda^{2} \frac{U}{M_{p}} P\left(M_{p}^{2}\right) & =C_{0} R\left(1-\frac{a_{1}}{2} R-\frac{a_{2}}{3} R^{2}-\frac{a_{3}}{4} R^{3}\right)+C_{1}-a_{1} C_{2} \\
\lambda^{2} M_{p} P\left(M_{p}^{2}\right) & =-B_{3} R^{2}\left(\frac{1}{2}-\frac{a_{1}^{\prime}}{3} R-\frac{a_{2}^{\prime}}{4} R^{2}\right)-B_{7}+a_{1}^{\prime} B_{9}
\end{aligned}
$$

the ratio of which yields $U$.

The choice of $R$ is dictated by stability considerations, it should be large enough to allow the use of the QCD expressions in the integrals over the circle of radius $R$ but not too large to invalidate the neglect of the integral over the continuum. The optimal value of $R$ is chosen in the stability region of both equations (35) and (36) Indeed, as can be seen in Fig. 1 both terms are practically constant for $1.5 \mathrm{GeV}^{2} \precsim R \precsim 3 \mathrm{GeV}^{2}$.

\section{$3 \quad$ Evaluation of the susceptibility $\chi$}

The next task is to calculate the susceptibility $\chi$ which enters in the expressions for the condensates. For this purpose I use a simple model independent method [11.

It follows from eq. 20 and from the Gell-Mann-Oakes-Renner relation [13]

$$
f_{\pi}^{2} m_{\pi}^{2}=-\left(m_{u}+m_{d}\right)\langle\bar{q} q\rangle
$$

that

$$
\chi=-\frac{\left(m_{u}+m_{d}\right)}{f_{\pi}^{2} m_{\pi}^{2}} \psi(0)
$$

with

$$
\psi\left(q^{2}\right)=i \int d^{4} x e^{i q x}\langle 0|T(\bar{q}(x) q(x))(\bar{q}(0) q(0))| 0\rangle
$$

This correlator exhibits a cut on the positive $t$ axis running from threshold to $\infty$ and consider a contour $C$ similar to the one used previously for the nucleon correlator. The integral over $C$ of the quantity $\psi(t) / t$ gives $\psi(0)$. As before the integration kernel is modified so as to minimize the contribution of the $0^{+}$ continuum, i.e. consider the integral

$$
\int_{C} d t \Delta(t) \psi(t)
$$




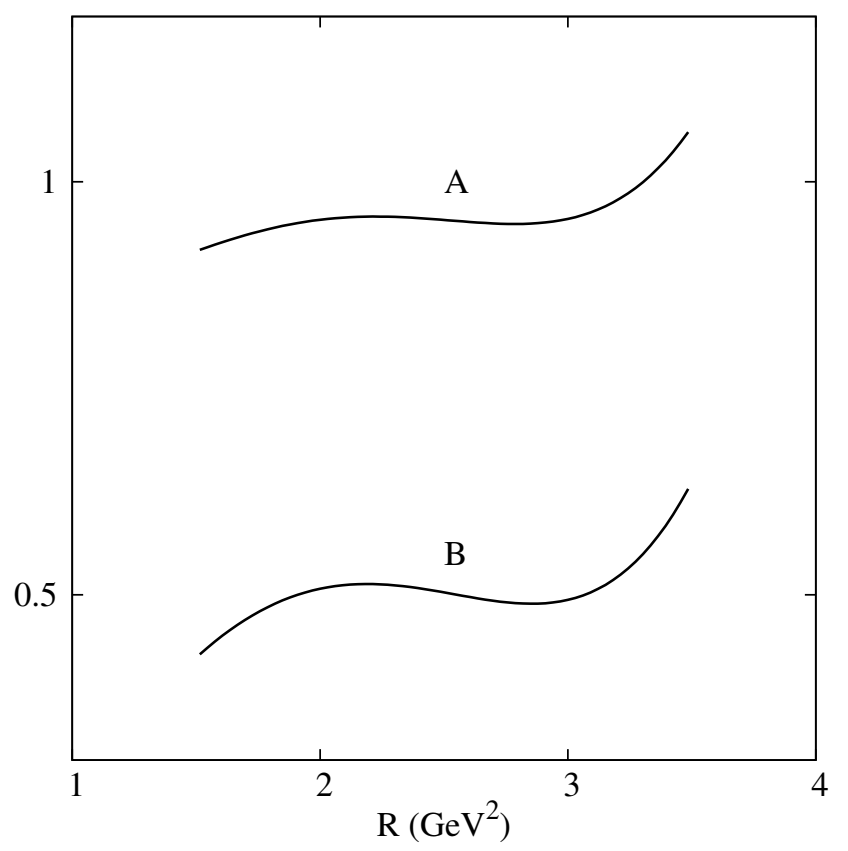

Figure 1: The right hand sides of (35) (A) and of (36)(B)as a function of $\mathrm{R}$ (not normalized) 


$$
\Delta(t)=\frac{1}{t}-b_{0}-b_{1} t
$$

we have

$$
\psi(0)=\frac{1}{\pi} \int_{c u t} d t \Delta(t) \psi(t)+\frac{1}{2 i \pi} \oint d t \Delta(t) \psi(t)
$$

In the first term on the r.h.s. of the equation above the integrand is impossible to model realistically and mostly unknown except for the existence of two isovector $0^{+}$resonances at $m_{1}^{2}=.97 \mathrm{GeV}^{2}$ and $m_{2}^{2}=2.10 \mathrm{GeV}^{2}$. It is expected that the overwhelming contribution to this integral over the cut is provided by the interval .90Gev $v^{2} \succsim \precsim 2.20 \mathrm{GeV}^{2}$

The choice of the constants $b_{0}$ and $b_{1}$ such that $\Delta\left(m_{1}^{2}\right)=\Delta\left(m_{2}^{2}\right)=0$ will practically annihilate the contribution of the integral over the continuum so that eq. (42) becomes

$$
\psi(0) \simeq \frac{1}{2 i \pi} \oint d t \psi_{Q C D}(t) \Delta(t)
$$

The perturbative part of the correlator eq. (43) is known to 5 loops in addition to 2 non-perturbative terms 12 .

$$
\psi_{Q C D}(t)=A_{0 .} t \ln (-t)+\frac{A_{1}}{t}+\frac{A_{2}}{t^{2}}+\ldots
$$

where

$$
\begin{gathered}
A_{0}=-\frac{3}{8 \pi^{2}}\left(1+\frac{11}{3} a+14.1785 a^{2}+77.3535 a^{3}+511.696 a^{4}+\ldots\right) \\
A_{1}=-\frac{1}{8}\langle a G G\rangle\left(1+\frac{16}{9} \cdot a\left(-1 G e V^{2}\right)+\frac{121}{18} a\right) \\
A_{2}=\frac{112}{27} \pi^{2} a\langle\bar{q} q\rangle^{2}
\end{gathered}
$$

So that eq. (43) gives

$$
\psi(0)=A_{0}\left(R-\frac{b_{0}}{2} R^{2}-\frac{b_{1}}{3} R^{3}\right)-b_{0} A_{1}-b_{1} A_{2}
$$

In the interval $1.5 \mathrm{GeV}^{2} \precsim R \precsim 2.5 \mathrm{GeV}^{2}$. the value of $\psi(0)$ oscillates between $-.30 \mathrm{GeV}$ and $-.35 \mathrm{GeV}$ which gives an estimate of the error involved and yields

$$
\chi=(1.03 \pm .10) \mathrm{GeV}^{-1}
$$

Which can be compared to values appearing in the litterature which lie in the range $.6 \mathrm{GeV}^{-1} \leq \chi \leq 3 \mathrm{GeV}^{-1}$.[Jin et al] 


\section{Results and Conclusions}

Before joining pieces together let us look at the values of the condensates which enter in the $A_{i}, B_{i}$ and $C_{i}$ :

$\langle\bar{q} q\rangle$ is obtained from the Gell-Mann, Oakes, Renner relation[13] with the values of the quark masses $m_{u}=(2.9 \pm .2) \mathrm{MeV}, m_{d}=(5.3 \pm .4) \mathrm{MeV}$ [14]

$$
4 \pi^{2}\langle\bar{q} q\rangle=-(.79 \pm .01) G e V^{3}
$$

The standard value is taken for the gluon condensate

$$
\langle a G G\rangle=(.012 \pm .006) G e V^{4}
$$

and

$$
\left\langle(\bar{q} q)^{2}\right\rangle=\kappa\langle\bar{q} q\rangle^{2}
$$

where $\kappa$ quantifies deviations from factorization.

We have finally

$U=\frac{M_{p}^{2}}{2}\left(C_{0} R\left(1-\frac{a_{1}}{2} R-\frac{a_{2}}{3} R^{2}-\frac{a_{3}}{4} R^{3}\right)+C_{1}-a_{1} C_{2}\right) /\left(-B_{3} R^{2}\left(\frac{1}{2}-\frac{a_{1}^{\prime}}{3} R-\frac{a_{2}^{\prime}}{4} R^{2}\right)-B_{7}-a_{1}^{\prime} B_{9}\right)$

Let us look at the sources of uncertainty in the expression above.

The first stems from the incomplete knowledge of the OPE: with our choice of polynomials the numerator of eq. (51) should be augmented by two additional terms $a_{2} C_{3}+a_{3} C_{4}$ and the denominator by $a_{2}^{\prime} B_{11}$. The higher order condensates $C_{3}, C_{4}$ and $B_{11}$ are of course unknown but it is possible to estimate them using the method of Pade' approximants. It turns out that the error introduced by the neglect of the higher order condensates amounts to no more than $2 \%$.

Another source of uncertainty stems from the choice of the coefficients $a_{1}^{\prime}, a_{2}^{\prime}$ (and consequently of the coefficients $a_{1}, a_{2}, a_{3}$ ), varying these within reasonable limit in order to deplete the contribution of the interval $2.0 \mathrm{GeV}^{2} \precsim t \precsim 3.0 \mathrm{GeV}^{2}$ introduces an uncertainty of $\sim 6-7 \%$ in the value of $U$.

An additional uncertainty of course is the one arising from the values of the condensates themselves.

All these uncertainties however are overwhelmed by the one coming from the undeterminacy in the value of $\kappa$ which measures the deviation from factorization in the value of the four quark condensate. There is no consensus on the value of this quantity in the litterature. Phenomenological estimates [15] place it in the range $1 \precsim \kappa \precsim 4$.

With a central value [14] $\delta m=2.4 \mathrm{MeV}, \delta M_{q}$ is plotted against $\kappa$ in Fig. 2 where the experimental limits are also shown.

Taking in consideration the uncertainty in $\delta m$ we see that the experimental value of $\delta M_{q}$ is reproduced for

$$
1.7 \lesssim \kappa \lesssim 2.3
$$




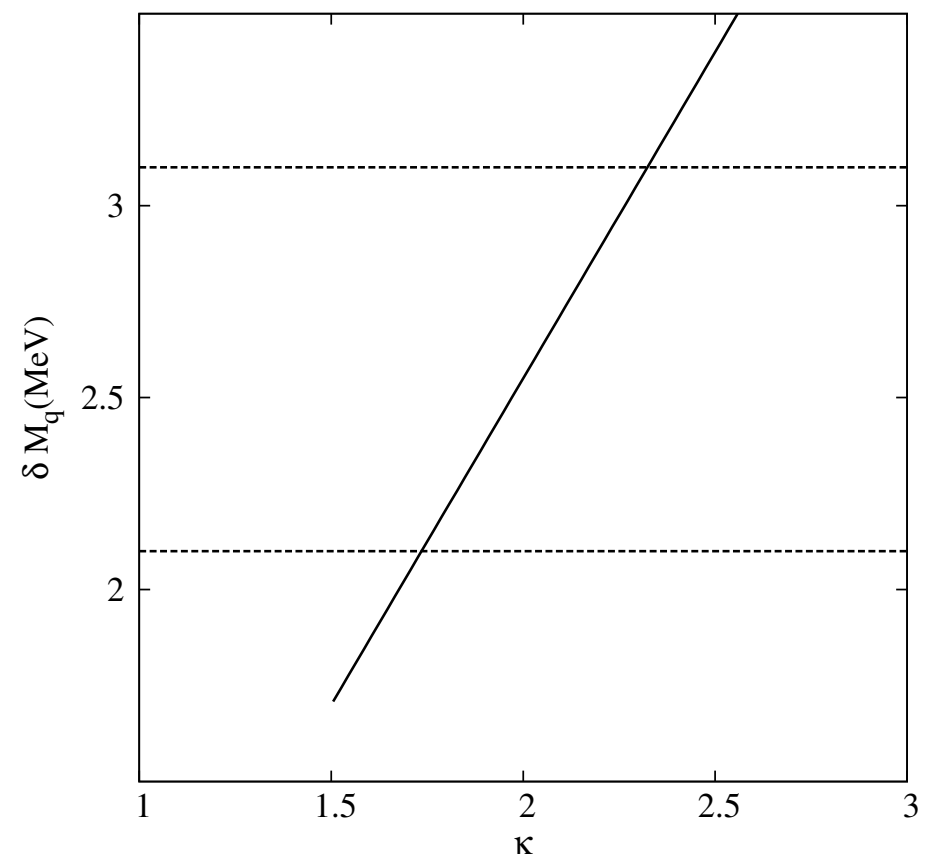

Figure 2: The strong part of the neutron-proton mass difference $\delta M_{q}$ as a function of $\kappa$ 


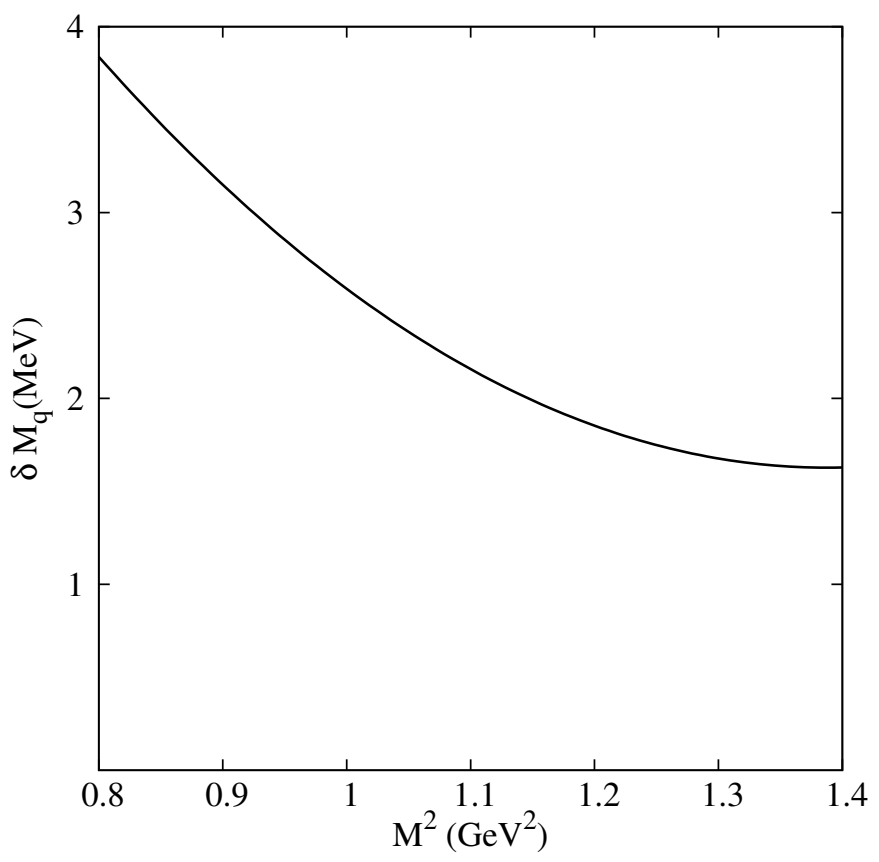

Figure 3: $\delta M_{q}$ as a function of the Borel mass $M^{2}$ for $\kappa=2$

It is instructive to compare the results obtained to the ones given by use of the exponential damping kernel $P(t)=e^{-\frac{t}{M^{2}}}$. Expression (51) for $U$ is then replaced by

$U=\frac{M_{p}^{2}}{2}\left(C_{0} M^{4} \int_{0}^{R / M^{2}} d x e^{-x}\left(x-\frac{M_{p}^{2}}{M^{2}}\right)-M_{p}^{2} C_{1}+\left(1+\frac{M_{p}^{2}}{M^{2}}\right) C_{2}\right) /\left(B_{3} M^{4} \int_{0}^{R / M^{2}} d x x e^{-x}+B_{7}-\frac{B_{9}}{M^{2}}\right)$

which reproduces qualitatively the same results as before but which fails to show good stability in variations of the Borel mass $M^{2}$ as can be seen from Fig. 3. This instability stems mostly from the fact that the contribution of the nucleon continuum remains important. In [4] the contribution of the single pole in eq.(24) is not eliminated but taken into account. This introduces an additional parameter and the accuracy of the method used to estimate it is hard to assess

To conclude, I have presented an evaluation of the neutron-proton mass difference $\delta M_{n p}$ using polynomial kernels in dispersion integrals taylored to reduce the contributions of the unknown parts of the continua to an extent which allows their neglect and which guarantees the stability of the calculation.

The numerical result depends sensitively on the value of $\kappa$ which quantifies the deviation of the value of the four quark condensate from the one given by 
the factorization assumption and reproduces the experimental value of $\delta M_{n p}$ for $\kappa \sim 2$. The old value $\delta M_{\text {coul }}=-(.76 \pm .30) M e V$ would correspond to values of $\kappa$ closer to unity.

It is finally worth to note that a recent analysis of Weinberg type sum rules [16] yields $\kappa=2.1_{-.2}^{+.3}$ 


\section{References}

[1] M. A. Shifman, A. I. Vainshtein and V. I. Zakharov, Nucl. Phys. B147, 385, 448 (1979)

[2] B. L. Ioffe, Nucl. Phys. B188 , 317 (1981) (E: B191 , 591(1981)); B. L. Ioffe and A. V. Smilga, Nuclear Physics B232 , 109 (1984)

[3] Y. Chung, H. G. Dosch, M. Kremer and D. Schall, Phys. Lett. 102B , 175 (1981); Y. Chung, H. G. Dosch, M. Kremer and D. Schall, Nucl. Phys. B197, 55 (1982)

[4] X. Jin, M. Nielsen and J. Pasupathy, Phys. Rev. D51, 3688 (1995); K-C Yang, W-Y. P. Hwang,E.M.Henley and L. S. Kisslinger, Phys. Rev. D47, 3001 (1993)

[5] M. Kremer, N.F. Nasrallah, N.A. Papadopoulos and K. Schilcher, Phys. Rev. D34, 2127 (1986), for recent applications of the method see e.g. references [11] and [14]

[6] W. N. Cottingham, Annals of Physics 25 , 424 (1963)

[7] J. Gasser and H. Leutwyler, Phys. Rep. 87 , 77 (1982)

[8] A. Walker-Loud, C. E. Carlsson and G. A. Miller, hep$\mathrm{ph} / 1203.0254$

[9] N. F. Nasrallah and K. Schilcher, Physics Letters B78 , 84 (1978) ; J.C. Collins, Nucl. Phys. B149 , 90 (1979)

[10] V. A. Sadovnikova, E. G. Drukarev, and M. G. Ryskin, Phys. Rev. D $72,114015(2005)$

[11] C. A. Dominguez, N. F. Nasrallah and K. Schilcher, J. High Energy Phys. 05,020 (2008)

[12] K. G. Chetyrkin and A. Khodjamirian, Eur. Phys. J. C46,721 (2006)

[13] M. Gell-Mann, R.J.Oakes and B. Renner, Physical Review 175,2195 (1968)

[14] C. A. Dominguez, N.F. Nasrallah, R. H. Rontsch, K. Schilcher, Phys. Rev. D79, 014009 (2009)

[15] K. Ackerstaff et al. (Opal collaboration), Eur. Phys. J. C7,571(1999) ; B. L. Ioffe and K. N. Zyablyuk, Nucl. Phys. A687,437 (2001) ; J. Bijnens, E. Gamiz and J. Prades, JHEP 0110,009 (2001) ; V. Cirigliano, E. Golowich and K. Maltman; Phys. Rev. D68 , 054013(2003); S. Friot, D. Greynat and E. de Rafael, JHEP 0410,043 (2004) ; S. Narison, Phys. Lett. B624,223 
(2005) ; M. Davier, A. Hocker and Z. Zhang, Rev. Mod. Phys. 78 , 1043 (2006); A. A. Almasy, K. Schilcher and H. Spiseberger, Eur.

Phys. J. C55,237 (2008) ; O. Cata, Phys. Rev. D81 ,054011 (2010)

[16] Paul Hohler and Ralf Rapp, hep-ph/1204.6309 